TITLE:

\title{
Trion formation and recombination dynamics in hole-doped single- walled carbon nanotubes
}

\section{AUTHOR(S):}

Nishihara, Taishi; Yamada, Yasuhiro; Okano, Makoto; Kanemitsu, Yoshihiko

\section{CITATION:}

Nishihara, Taishi ...[et al]. Trion formation and recombination dynamics in hole-doped single-walled carbon nanotubes. Applied Physics Letters 2013, 103(2): 023101.

ISSUE DATE:

2013-07

URL:

http://hdl.handle.net/2433/176986

RIGHT:

(c) 2013 AIP Publishing LLC 


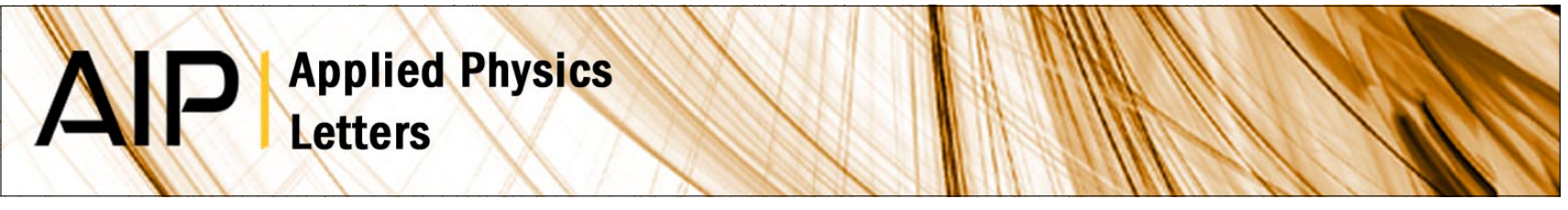

Trion formation and recombination dynamics in hole-doped single-walled carbon nanotubes

Taishi Nishihara, Yasuhiro Yamada, Makoto Okano, and Yoshihiko Kanemitsu

Citation: Appl. Phys. Lett. 103, 023101 (2013); doi: 10.1063/1.4813014

View online: http://dx.doi.org/10.1063/1.4813014

View Table of Contents: http://apl.aip.org/resource/1/APPLAB/v103/i2

Published by the AIP Publishing LLC.

Additional information on Appl. Phys. Lett.

Journal Homepage: http://apl.aip.org/

Journal Information: http://apl.aip.org/about/about_the_journal

Top downloads: http://apl.aip.org/features/most_downloaded

Information for Authors: http://apl.aip.org/authors

\section{ADVERTISEMENT}
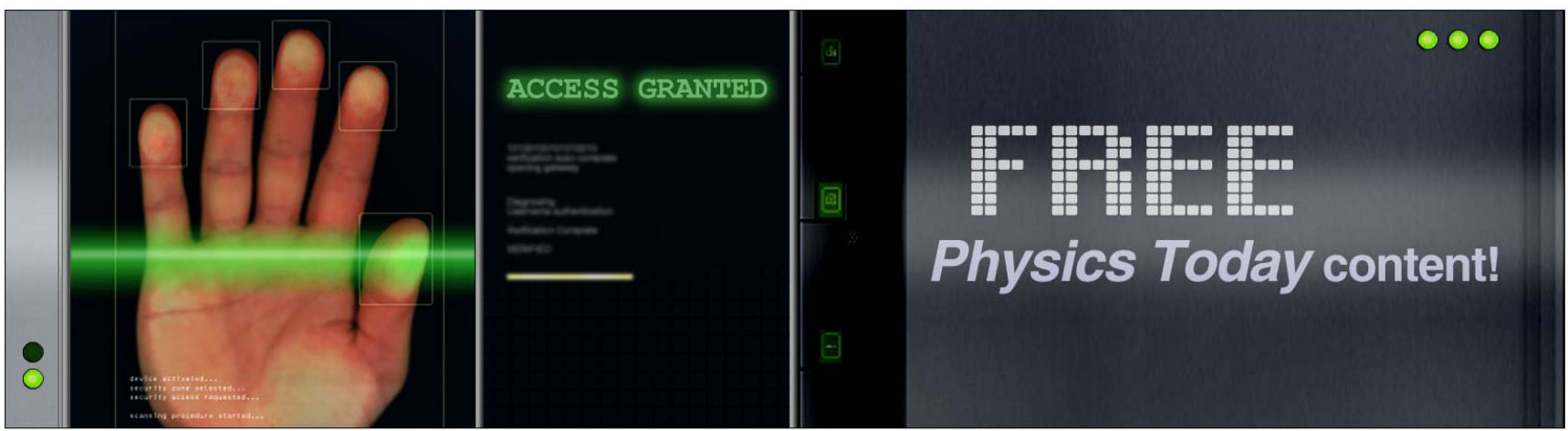


\title{
Trion formation and recombination dynamics in hole-doped single-walled carbon nanotubes
}

\author{
Taishi Nishihara, Yasuhiro Yamada, Makoto Okano, and Yoshihiko Kanemitsu ${ }^{\text {a) }}$ \\ Institute for Chemical Research, Kyoto University, Uji, Kyoto 611-0011, Japan
}

(Received 12 April 2013; accepted 17 June 2013; published online 8 July 2013)

\begin{abstract}
We studied the trion (charged exciton) formation and recombination dynamics in hole-doped $(7,5)$ single-walled carbon nanotubes (SWCNTs) by performing femtosecond transient absorption spectroscopy. The doping of SWCNTs with holes leads to a fast decay component from an exciton to a trion, and the trion decays with a lifetime of a few picoseconds. The experimental results can be explained by a quantized model accounting for the dark exciton and trion states and the hole number distribution in a SWCNT. Our findings show that the optical responses of SWCNTs can be manipulated by doping of SWCNTs with a small number of holes. (C) 2013 AIP Publishing LLC.

[http://dx.doi.org/10.1063/1.4813014]
\end{abstract}

Over the past decade, the optical properties of semiconducting single-walled carbon nanotubes (SWCNTs) have been studied extensively from the viewpoints of fundamental physics and potential applications in various devices. SWCNTs have one-dimensional (1D) nanostructures in which strong Coulomb interactions between electrons and holes produce stable excitons. These excitons govern the optical properties of SWCNTs even at room temperature, because SWCNTs have extremely large exciton binding energies. ${ }^{1-5}$ Various unique and interesting 1D-exciton-related optical processes have been observed, such as quantized excitonAuger recombination (exciton-exciton annihilation) and multiple exciton generation. ${ }^{6-15}$ Moreover, SWCNTs have complicated fine structures near the lowest exciton state because of their unique structures. ${ }^{16,17}$ Recent magnetoluminescence studies of a single SWCNT revealed the existence of dipole-forbidden dark exciton states below the lowest-energy dipole-allowed bright excitons. ${ }^{18,19}$ This underlying optically inactive dark exciton affects the optical properties of the SWCNTs determined by the optically active bright exciton. ${ }^{20}$ However, the interplay between the bright and dark excitons in terms of the dynamics are complicated and remain unclear, ${ }^{21,22}$ and the impact of dark excitons on the optical responses of SWCNTs is also unclear.

The optical properties of SWCNTs are sensitive to carrier doping. ${ }^{23-32}$ Drastic changes in the absorption and photoluminescence due to the bright excitons have been observed as the doped hole density increases. ${ }^{24,28}$ Because of the conservation of energy and momentum, charge carriers can interact effectively with dark excitons, ${ }^{33}$ and carrier doping could modify the optical responses of SWCNTs through these dark excitons. Moreover, positive trions (positively charged excitons) have recently been discovered in holedoped SWCNTs at room temperature. ${ }^{28}$ The trion state is located energetically below the dark exciton state. These trions can strongly affect the decay dynamics of the brightand dark-exciton populations in hole-doped SWCNTs. Therefore, experimental studies of the temporal change in the trion populations can provide a clear understanding of

\footnotetext{
${ }^{\text {a) }}$ Author to whom correspondence should be addressed. Electronic mail: kanemitu@scl.kyoto-u.ac.jp
}

the global features of excitons, including bright and dark excitons, in SWCNTs doped with carriers.

In this work, we study the trion and exciton recombination dynamics in hole-doped SWCNTs using pump-probe transient absorption (TA) spectroscopy. The bright $E_{11}$ exciton decay is faster in hole-doped SWCNTs than in undoped SWCNTs. Trion decay with a lifetime of a few picoseconds is observed under resonant excitation of trions. We propose a simple model taking account of the dark exciton level and the number of holes in a SWCNT, and this model successfully reproduces the experimental results on the bright $E_{11}$ exciton decay. Our model indicates that hole doping creates a relaxation channel from the bright exciton to the trion through the dark exciton.

Carbon nanotubes were synthesized using the CoMoCAT methods and dispersed in toluene solutions with 0.5 wt. $\%$ poly[9,9-dioctylfluorenyl-2,7-diyl] (PFO). ${ }^{34}$ The samples were treated as follows: moderate bath sonication for $60 \mathrm{~min}$, vigorous sonication with a tip-type sonicator for $5 \mathrm{~h}$, and ultracentrifugation at $18500 \mathrm{~g}$ for $90 \mathrm{~min}$. Hole doping was achieved using a p-type dopant, 2,3,5,6-tetrafluoro7,7,8,8-tetracyanoquinodimethane $\left(\mathrm{F}_{4} \mathrm{TCNQ}\right){ }^{26}$ Transient absorption spectroscopy was performed using a pump-probe technique. A quartz sample cell with a path length of $1 \mathrm{~mm}$ was used for the TA measurements. The light source was a wavelength-tunable optical parametric amplifier based on a regenerative amplified mode-locked Ti:sapphire laser with a pulse duration of $150 \mathrm{fs}$ and a repetition rate of $1 \mathrm{kHz}$. The laser spot size on the sample surface was measured carefully using the knife-edge method. All measurements were performed at room temperature.

Figure 1 shows the absorption spectra of undoped and hole-doped SWCNT samples. For the undoped samples, a strong peak is observed at $1.18 \mathrm{eV}$, which is attributed to the $E_{11}$ bright exciton for $(7,5)$ SWCNTs. ${ }^{34}$ Thus, the absorption spectrum shows that our samples are highly enriched in $(7,5)$ chirality. Hole doping markedly reduces the bright exciton intensity at the $1.18 \mathrm{eV}$ absorption peak. In the hole-doped sample, a absorption peak appears at $1.02 \mathrm{eV}$, which is assigned to the trion absorption for $(7,5)$ SWCNTs. ${ }^{28}$ Enlarged spectra around the trion absorption for $(7,5)$ SWCNTs are shown in the inset. For undoped samples, a 


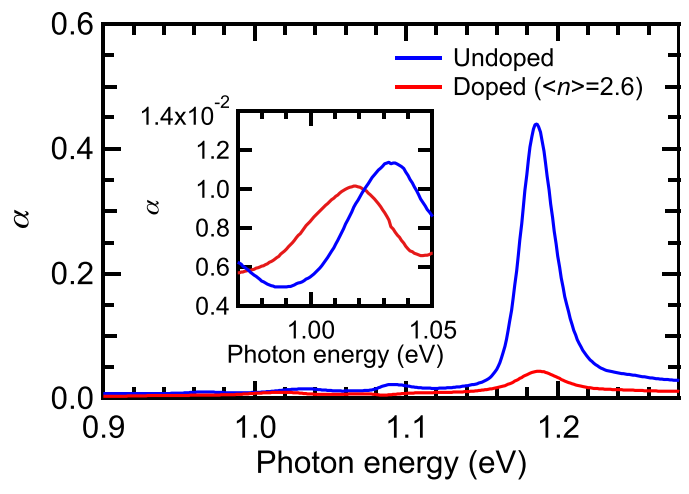

FIG. 1. Optical absorption spectra of undoped and hole-doped single-walled carbon nanotubes. The inset shows an enlarged view of the lower-energy region.

weak absorption peak is observed at $1.04 \mathrm{eV}$, which is attributed to the bright $E_{11}$ exciton for $(8,6)$ SWCNTs. ${ }^{34}$ Note that the $(8,6)$ SWCNT bright exciton peak disappears after holedoping because larger-diameter SWCNTs are more susceptible to the hole-doping effects than small-diameter SWCNTs. ${ }^{26}$ Thus, the $(8,6)$ SWCNTs have no impact on the optical responses in hole-doped samples.

To clarify the effects of hole doping on the optical responses of SWCNTs, we first studied the $E_{11}$ bright exciton decay dynamics of hole-doped SWCNTs. With increasing hole-dopant concentration, the optical absorption intensity of the bright exciton decreases but that of the trion increases: there is an anticorrelation between the exciton and trion intensities. $^{28}$ Therefore, under our experimental conditions, the sample with a certain dopant concentration (Fig. 1) is good for measurements of both the exciton and trion decay dynamics. Figure 2(a) shows the TA decay curves of undoped and hole-doped samples, where the probe and pump energies were tuned to the $E_{11}$ exciton state for $(7,5)$ SWCNTs. The TA decay curves are normalized at the maximum values. The photoinduced absorption change $\Delta \alpha$ is negative because of the photobleaching of the $E_{11}$ exciton state. Both undoped and hole-doped samples show nonexponential TA decay curves. After hole doping, a fast-decay component clearly appears, indicating that hole doping creates additional relaxation channels due to exciton-hole interactions. The most plausible mechanism of these hole-induced relaxation channels is trion formation, as suggested by the correlation between the presence of the trion peak and the appearance of the fast-decay component. Therefore, we next analyze the exciton decay dynamics in hole-doped SWCNTs using a simple model that includes the trion state.

For this model, we consider the exciton and trion energy states in hole-doped SWCNTs. It has been experimentally verified that the dark exciton states exist below the lowestenergy bright exciton state. ${ }^{18}$ Recent studies revealed that the trion state is energetically lower than the dark exciton state and that the lowest-energy trion is the bright trion, which is composed of the dark exciton and the carrier. ${ }^{35}$ This indicates that the relaxation process from the bright exciton to the trion will occur through the dark exciton state. Thus, we consider a simple four-level model including the ground, bright exciton, dark exciton, and trion states for hole-doped SWCNTs, as illustrated in Fig. 2(b). In this four-level model, (a)

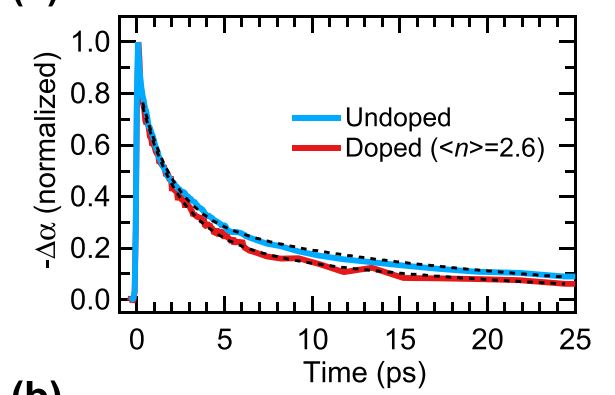

(b)

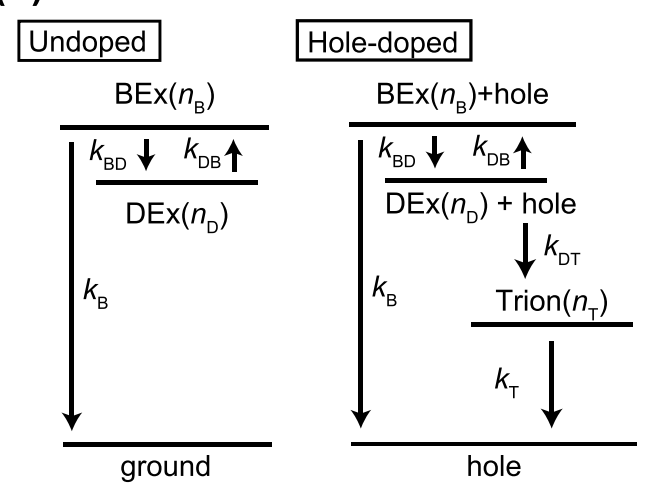

(c)

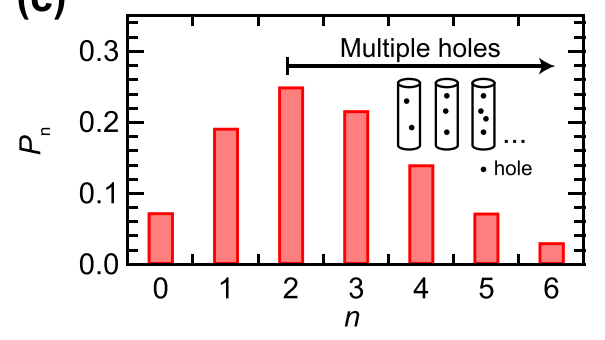

FIG. 2. (a) TA decay curves for the $E_{11}$ excitons in the undoped and holedoped samples. The dotted curves are the fitting curves. (b) Kinetic diagram accounting for the four energy levels. (c) The probability of finding SWCNTs having the $n$ holes, $P_{\mathrm{n}}$, for the sample of an average hole number $\langle n\rangle$ of 2.6 .

dark excitons interact strongly with doped holes. Thus, the rate equations are given by

$$
\begin{aligned}
& \frac{d n_{\mathrm{B}}}{d t}=-\left(k_{\mathrm{B}}+k_{\mathrm{BD}}\right) n_{\mathrm{B}}+k_{\mathrm{DB}} n_{\mathrm{D}}, \\
& \frac{d n_{\mathrm{D}}}{d t}=-\left(k_{\mathrm{DB}}+k_{\mathrm{DT}}\right) n_{\mathrm{D}}+k_{\mathrm{BD}} n_{\mathrm{B}}, \\
& \frac{d n_{\mathrm{T}}}{d t}=-k_{\mathrm{T}} n_{\mathrm{T}}+k_{\mathrm{DT}} n_{\mathrm{D}},
\end{aligned}
$$

where $n_{\mathrm{B}}, n_{\mathrm{D}}$, and $n_{\mathrm{T}}$ are the populations of bright excitons, dark excitons, and trions. The coefficients $k_{\mathrm{B}}$ and $k_{\mathrm{BD}}$ correspond to the decay rates from the bright exciton state to the ground state and the dark exciton state, respectively. In addition, $k_{\mathrm{DB}}$ corresponds to the transition rate from the dark exciton to the bright exciton states, and $k_{\mathrm{DT}}$ and $k_{\mathrm{T}}$ are the decay rates from the dark excitons to the trions and from the trions to the ground state, respectively. Assuming that the transition between the bright and dark exciton states occurs via one-phonon absorption and emission processes, $k_{\mathrm{BD}}$ and $k_{\mathrm{DB}}$ can be represented as $\gamma\left(n_{\mathrm{p}}+1\right)$ and $\gamma n_{\mathrm{p}}$, respectively, where $n_{\mathrm{p}}=1 /[\exp (\Delta E / k T)-1]$ is the phonon occupation number, $\gamma$ is the temperature-independent scattering 
rate, and $k$ is Boltzmann factor. ${ }^{20,22}$ The energy splitting between the dark and bright excitons $\Delta E$ is about $4 \mathrm{meV}$ in $(7,5)$ SWCNTs, ${ }^{18}$ which confirms that an approximation of $k_{\mathrm{BD}} \approx k_{\mathrm{DB}}$ is valid at room temperature.

On the basis of Eq. (1), we fitted the $E_{11}$ exciton dynamics for the undoped SWCNT sample. Here, we set $k_{\mathrm{DT}}=0$, because no trions are formed by photoexcitation in the undoped SWCNTs. The fitting result is shown in Fig. 2(a) as a dotted curve that reproduces the experimental data well. The best-fitting parameters are $k_{\mathrm{B}}{ }^{-1}=8.3 \mathrm{ps}$ and $k_{\mathrm{BD}}{ }^{-1}=3.8 \mathrm{ps}$, which are quite close to the previously reported values. ${ }^{10}$

In hole-doped samples, the TA decay curves exhibit a fast-decaying component that is not observed in undoped samples. However, the TA decay curves show almost no hole-density dependence after $20 \mathrm{ps}$. These results strongly indicate that the hole-doped sample is a mixture of undoped and doped SWCNTs with different hole numbers and that the fast-decaying TA component corresponds to the exciton decay dynamics of the hole-doped SWCNTs. ${ }^{29}$ We fitted the fast-decaying TA component with Eq. (1), where we assumed that the bright-dark transition rate is unchanged by the hole doping, i.e., $k_{\mathrm{BD}}{ }^{-1}=3.8 \mathrm{ps}$. We calculated the bestfitting parameters as $k_{\mathrm{B}}{ }^{-1}=6.6 \mathrm{ps}$ and $k_{\mathrm{DT}}{ }^{-1}=2.7 \mathrm{ps}$, and the calculated TA curve for the hole-doped sample is shown in Fig. 2(a) as a dotted curve. The recombination rate for the bright exciton, $k_{\mathrm{B}}$, is enhanced by the hole doping, which indicates that hole-doping induces a rapid process for the decay of bright excitons such as exciton-hole Auger recombination. ${ }^{33}$

For quantitative discussions on the TA decay curves, we need to consider the number of holes in SWCNTs. Because of the 1D motion of excitons in hole-doped SWCNTs, the exciton-hole scattering occurs between an exciton and the nearest holes. The exciton decay rate will be sensitive to the number of hole within the exciton excursion range, in which the motion of exciton is spatially limited. ${ }^{27}$ First, we assume that the probability that $n$ holes are found within the exciton excursion range, $P_{n}$, is given by the Poisson distribution, $P_{n}=\langle n\rangle^{n} \mathrm{e}^{-\langle n\rangle} / n$ !, where $\langle n\rangle$ is the average hole number in the exciton excursion range. Based on the hole-number dependence of the absorption coefficient (the absorption of the excitons decreases exponentially with the hole number), ${ }^{29}$ the value of $\langle n\rangle$ for the hole-doped SWCNT sample was estimated to be 2.6. The probability of $n$-holes being inside exciton excursion range in this sample is illustrated in Fig. 2(c). In the following, we use the expression a $n$-holes doped SWCNT to define a SWCNT with $n$-holes within the exciton excursion range. These results suggest that our sample contains doped SWCNTs with two holes or more and that the probability of finding undoped or one-hole-doped SWCNTs is below 30\%. However, it is known that the exciton absorption intensity is markedly quenched by hole doping. ${ }^{26,28,29}$ In particular, assuming a Poisson distribution for the number of doped holes and given the absorption intensity ratios, ${ }^{29}$ the contribution of the undoped and one-hole-doped SWCNTs to the exciton absorption is estimated to be $75 \%$ or more in our sample. Therefore, the ensemble-averaged exciton absorption signals of our hole-doped samples are dominantly due to exciton absorption for the undoped and one-hole-doped SWCNTs. Therefore, we conclude that the fast-decaying TA component from our hole-doped sample is mainly contributed by the SWCNTs doped with one hole.

To gain a deep understanding of the exciton recombination dynamics in the hole-doped SWCNTs, we studied the trion formation and recombination dynamics under resonant trion or bright exciton excitation. Figure 3(a) shows the TA decay dynamics of the hole-doped sample probed at energies of $1.00,1.02$, and $1.04 \mathrm{eV}$. The pump energy is $1.02 \mathrm{eV}$, which is tuned to the energy of the trions for $(7,5)$ nanotubes. All the TA signals show a negative photoinduced absorption change $\Delta \alpha$ and decays within a few picoseconds. At around $t=0$, the TA curves have an oscillating component that is likely due to coherent artifacts.

In Fig. 3(b), we plot the TA spectra, i.e., the probeenergy dependence of the TA intensity. The solid curves are guides to the eye, and the absorption spectrum of the holedoped sample is shown as a dotted curve in the same figure. At a delay time of $1 \mathrm{ps}$, the TA spectrum peaks at $1.01 \mathrm{eV}$ and is similar to the absorption spectrum. Note that no photobleaching signal is observed at $1.01 \mathrm{eV}$ for the undoped SWCNT sample. On the basis of these observations, we conclude that the TA signals at around $1.01 \mathrm{eV}$ originate from the trions for the hole-doped $(7,5)$ SWCNTs. The initial rise in the TA signal occurs within a pulse width, indicating that trions are directly generated by the pump excitation at $1.02 \mathrm{eV}$. We fitted the TA dynamics with a single exponential function in the range between 0.3 and $10 \mathrm{ps}$, as shown in Fig. 3(a) with dotted curves. The relaxation time was estimated to be $1.5 \mathrm{ps}$, which is independent of the probe energy. Therefore, we successfully determined the trion lifetime $k_{\mathrm{T}}^{-1}$ to be $1.5 \mathrm{ps}$.

The process for the formation of the trion from the $E_{11}$ bright exciton was also studied under resonant excitation of the bright exciton, where the pump and probe energies were tuned to $E_{11}$ bright excitons and trions for $(7,5)$ nanotubes, respectively, as shown in Fig. 3(c). The TA signal rapidly increases after pump excitation. We fitted the TA dynamics using rate Eq. (1), where we fixed $k_{\mathrm{BD}}{ }^{-1}=3.8 \mathrm{ps}$. In result,
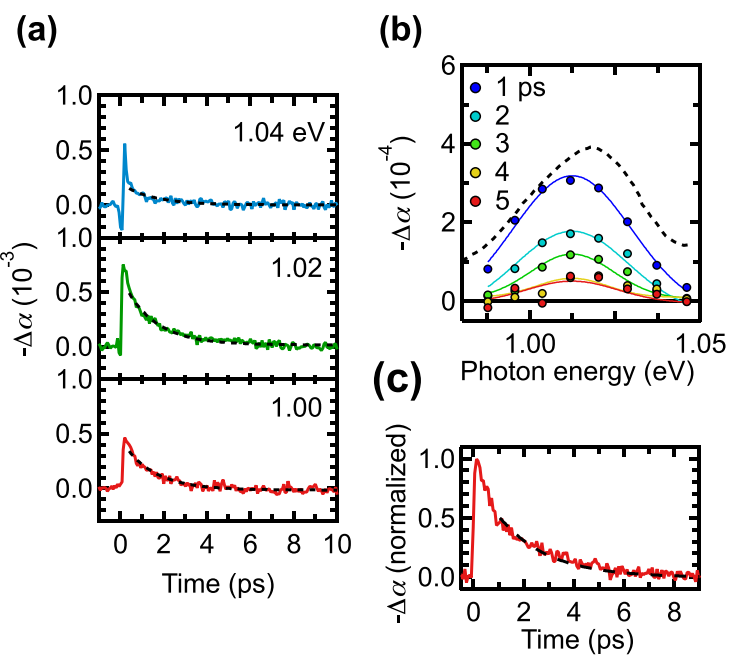

FIG. 3. (a) TA decay curves of the hole-doped sample probed at 1.00, 1.02, and $1.04 \mathrm{eV}$. (b) TA spectra at different delay times. The solid lines are guides to the eye. The dotted line is the absorption spectrum of the holedoped sample. (c) TA decay curve probed at $1.02 \mathrm{eV}$ under excitation of the $E_{11}$ exciton. 
we obtained $k_{\mathrm{T}}{ }^{-1}=1.9 \mathrm{ps}, k_{\mathrm{DT}}{ }^{-1}=150 \mathrm{fs}$, and $k_{\mathrm{B}}{ }^{-1}=170 \mathrm{fs}$ as the best-fitting parameters. The trion decay rate $k_{\mathrm{T}}$ shows good agreement with that estimated from the trion decay dynamics under resonant trion excitation. On the other hand, the dark exciton-trion transition rate $k_{\mathrm{DT}}$ and the bright exciton decay rate $k_{\mathrm{B}}$ are much larger than that estimated from the $E_{11}$ exciton decay dynamics of hole-doped sample. To resolve this discrepancy, we need to consider the holenumber distribution of the hole-doped samples.

As mentioned above, the TA signal of the hole-doped sample probed at the bright exciton energy is mainly determined by the TA signal from the undoped and one-holedoped SWCNTs, because the exciton absorption decreases with increasing hole number. On the other hand, the TA signal probed at the trion energy is determined by the SWCNTs with multiple holes, because the trion absorption intensity increases with hole number. ${ }^{29}$ Therefore, the trion-probed TA signals reflect the temporal changes in the trion populations in the multiple-hole-doped SWCNTs rather than in the one-hole-doped SWCNTs. The hole number distribution in the sample causes the discrepancy of the ensemble-averaged decay rates. As a result, the values of $k_{\mathrm{DT}}$ and $k_{\mathrm{B}}$ estimated from the trion decay are larger than those estimated from the exciton decay in the ensemble sample, because the $k_{\mathrm{DT}}$ and $k_{\mathrm{B}}$ increase with hole number through the exciton-hole interaction in a single SWCNT. Thus, by considering the hole number distribution, we revealed the exciton and trion decay processes in the hole-doped SWCNTs.

In conclusion, we studied the exciton and trion recombination dynamics in undoped and hole-doped SWCNTs by performing TA spectroscopy using a pump-probe technique. The $E_{11}$ exciton decay in the hole-doped SWCNT sample is shorter than that in the undoped SWCNT sample, and trion decay with a lifetime of a few picoseconds is observed. We proposed a model with four energy levels accounting for the dark exciton level, and this model reproduced the experimental results. Our model also indicates that the hole doping creates a relaxation channel from dark excitons to trions.

Part of this work was supported by KAKENHI (20104006, 25247052), MEXT Project of Integrated Research on Chemical Synthesis, The Sumitomo Electric Industries Group CSR Foundation, and JST-CREST.

${ }^{1}$ T. Ando, J. Phys. Soc. Jpn. 66, 1066 (1997).

${ }^{2}$ M. S. Dresselhaus, G. Dresselhaus, R. Saito, and A. Jorio, Annu. Rev. Phys. Chem. 58, 719 (2007).

${ }^{3}$ Y. Kanemitsu, Phys. Chem. Chem. Phys. 13, 14879 (2011).
${ }^{4}$ F. Wang, G. Dukovic, L. E. Brus, and T. F. Heinz, Science 308, 838 (2005).

${ }^{5}$ J. Maultzsch, R. Pomraenke, S. Reich, E. Chang, D. Prezzi, A. Ruini, E. Molinari, M. S. Strano, C. Thomsen, and C. Lienau, Phys. Rev. B 72, 241402 (2005).

${ }^{6}$ F. Wang, G. Dukovic, E. Knoesel, L. E. Brus, and T. F. Heinz, Phys. Rev. B 70, 241403 (2004).

${ }^{7}$ L. Valkunas, Y.-Z. Ma, and G. R. Fleming, Phys. Rev. B 73, 115432 (2006).

${ }^{8}$ L. Huang and T. D. Krauss, Phys. Rev. Lett. 96, 057407 (2006).

${ }^{9}$ R. M. Russo, E. J. Mele, C. L. Kane, I. V. Rubtsov, M. J. Therien, and D. E. Luzzi, Phys. Rev. B 74, 041405 (2006).

${ }^{10}$ Z. Zhu, J. Crochet, M. S. Arnold, M. C. Hersam, H. Ulbricht, D. Resasco, and T. Hertel, J. Phys. Chem. C 111, 3831 (2007).

${ }^{11}$ A. Ueda, K. Matsuda, T. Tayagaki, and Y. Kanemitsu, Appl. Phys. Lett. 92, 233105 (2008).

${ }^{12}$ L. Lüer, S. Hoseinkhani, D. Polli, J. Crochet, T. Hertel, and G. Lanzani, Nat. Phys. 5, 54 (2009).

${ }^{13}$ Y. Murakami and J. Kono, Phys. Rev. Lett. 102, 037401 (2009).

${ }^{14}$ S. Wang, M. Khafizov, X. Tu, M. Zheng, and T. D. Krauss, Nano Lett. 10, 2381 (2010).

${ }^{15}$ Y. Kanemitsu, Acc. Chem. Res. 46, 1358 (2013).

${ }^{16}$ S. Zaric, G. N. Ostojic, J. Kono, J. Shaver, V. C. Moore, M. S. Strano, R. H. Hauge, R. E. Smalley, and X. Wei, Science 304, 1129 (2004).

${ }^{17}$ I. B. Mortimer and R. J. Nicholas, Phys. Rev. Lett. 98, 027404 (2007).

${ }^{18}$ R. Matsunaga, K. Matsuda, and Y. Kanemitsu, Phys. Rev. Lett. 101, 147404 (2008).

${ }^{19}$ A. Srivastava, H. Htoon, V. I. Klimov, and J. Kono, Phys. Rev. Lett. 101, 087402 (2008).

${ }^{20}$ R. Matsunaga, Y. Miyauchi, K. Matsuda, and Y. Kanemitsu, Phys. Rev. B 80, 115436 (2009).

${ }^{21}$ M. Jones, W. K. Metzger, T. J. McDonald, C. Engtrakul, R. J. Ellingson, G. Rumbles, and M. J. Heben, Nano Lett. 7, 300 (2007).

${ }^{22}$ S. Berciaud, L. Cognet, and B. Lounis, Phys. Rev. Lett. 101, 077402 (2008).

${ }^{23}$ M. S. Strano, C. B. Huffman, V. C. Moore, M. J. O'Connell, E. H. Haroz, J. Hubbard, M. Miller, K. Rialon, C. Kittrell, S. Ramesh, R. H. Hauge, and R. E. Smalley, J. Phys. Chem. B 107, 6979 (2003).

${ }^{24}$ G. N. Ostojic, S. Zaric, J. Kono, M. S. Strano, V. C. Moore, R. H. Hauge, and R. E. Smalley, Phys. Rev. Lett. 92, 117402 (2004).

${ }^{25}$ G. Dukovic, B. E. White, Z. Zhou, F. Wang, S. Jockusch, M. L. Steigerwald, T. F. Heinz, R. A. Friesner, N. J. Turro, and L. E. Brus, J. Am. Chem. Soc. 126, 15269 (2004).

${ }^{26}$ M. O'Connell, E. E. Eibergen, and S. K. Doorn, Nat. Mater. 4, 412 (2005).

${ }^{27}$ L. Cognet, D. A. Tsyboulski, J.-D. R. Rocha, C. D. Doyle, J. M. Tour, and R. B. Weisman, Science 316, 1465 (2007).

${ }^{28}$ R. Matsunaga, K. Matsuda, and Y. Kanemitsu, Phys. Rev. Lett. 106, 037404 (2011).

${ }^{29}$ T. Nishihara, Y. Yamada, and Y. Kanemitsu, Phys. Rev. B 86, 075449 (2012).

${ }^{30}$ S. Yasukochi, T. Murai, S. Moritsubo, T. Shimada, S. Chiashi, S. Maruyama, and Y. K. Kato, Phys. Rev. B 84, 121409 (2011).

${ }^{31}$ T. Koyama, S. Shimizu, Y. Miyata, H. Shinohara, and A. Nakamura, Phys. Rev. B 87, 165430 (2013).

${ }^{32}$ Y. Kimoto, M. Okano, and Y. Kanemitsu, Phys. Rev. B 87, 195416 (2013).

${ }^{33}$ J. M. Kinder and E. J. Mele, Phys. Rev. B 78, 155429 (2008).

${ }^{34}$ A. Nish, J.-Y. Hwang, J. Doig, and R. J. Nicholas, Nat. Nanotechnol. 2, 640 (2007).

${ }^{35}$ K. Watanabe and K. Asano, Phys. Rev. B 85, 035416 (2012). 